\title{
An accelerometer-based navigation system provides acetabular cup orientation accuracy comparable to that of computed tomography-based navigation during total hip arthroplasty in the supine position
}

Tomonori Tetsunaga ${ }^{1 *}$, Kazuki Yamada $^{2}$, Tomoko Tetsunaga ${ }^{1}$, Tomoaki Sanki ${ }^{1}$, Yoshi Kawamura ${ }^{1}$ and Toshifumi Ozaki ${ }^{1}$

\begin{abstract}
Background: Inadequate acetabular component orientation is associated with postoperative impingement, dislocation, and accelerated polyethylene wear. Computed tomography (CT)-based navigation systems provide accuracy for total hip arthroplasty (THA) but are not available in all facilities. Accelerometer-based navigation systems are inexpensive, but their accuracy remains undetermined. This study compares the accuracy of cup orientation in THA using CT-based and accelerometer-based navigation systems.

Methods: This retrospective study included 35 consecutive patients (11 males, 24 females; mean age, 65 years) who underwent primary cementless THA via an anterolateral approach in the supine position. Both CT-based and accelerometer-based navigation systems were used simultaneously. The accuracy of cup orientation was compared between the two systems using postoperative CT.

Results: The accuracy of cup inclination was $2.7^{\circ} \pm 2.0^{\circ}$ in the CT-based group and $3.3^{\circ} \pm 2.4^{\circ}$ in the accelerometer-based group. The accuracy of cup anteversion was $2.8^{\circ} \pm 2.6^{\circ}$ in the $C$-based group and $3.4^{\circ} \pm 2.2^{\circ}$ in the accelerometer-based group. No significant difference was observed in cup inclination $(p=0.29)$ or cup anteversion $(p=0.34)$ between CT-based and accelerometer-based navigation.
\end{abstract}

Conclusions: The accuracy of cup positioning did not differ significantly between CT-based and accelerometer-based navigation systems.

Keywords: Hip, Navigation system, Total hip replacement, Retrospective study

\footnotetext{
*Correspondence: tomonori_t31@yahoo.co.jp

'Department of Orthopaedic Surgery, Okayama University Hospital, 2-5-1

Shikata-cho, Kitaku, Okayama 700-8558, Japan

Full list of author information is available at the end of the article
}

(c) The Author(s). 2020 Open Access This article is licensed under a Creative Commons Attribution 4.0 International License, which permits use, sharing, adaptation, distribution and reproduction in any medium or format, as long as you give appropriate credit to the original author(s) and the source, provide a link to the Creative Commons licence, and indicate if changes were made. The images or other third party material in this article are included in the article's Creative Commons licence, unless indicated otherwise in a credit line to the material. If material is not included in the article's Creative Commons licence and your intended use is not permitted by statutory regulation or exceeds the permitted use, you will need to obtain permission directly from the copyright holder. To view a copy of this licence, visit http://creativecommons.org/licenses/by/4.0/ The Creative Commons Public Domain Dedication waiver (http://creativecommons.org/publicdomain/zero/1.0/) applies to the data made available in this article, unless otherwise stated in a credit line to the data. 


\section{Background}

Correct implant positioning is important for good longterm results in total hip arthroplasty (THA). Inadequate orientation of the acetabular component is associated with postoperative impingement [1], dislocation [2], accelerated polyethylene wear [3], liner damage, and restricted range of motion [4]. Surgeons use intraoperative navigation systems to improve the accuracy of implant orientation [5-7]. Computed tomography (CT)-based navigation systems that match preoperative images with the actual pelvis use different techniques. These include CT-based 2-dimensional (2D)-3D matching navigation using intraoperative fluoroscopy, CT-based paired-point matching navigation based on the identification and matching of anatomical landmarks, and surface matching using random identification and registration of reference points [8]. These navigation systems are capable of displaying the angle and position in real time, further improving placement accuracy over CT-free navigation and reducing the rates of dislocation, impingement, and revision THA compared to those of conventional methods [9]. However, CT-based navigation systems also have some disadvantages. 2D-3D matching requires radiation exposure because multidirectional fluoroscopic images must be matched with 3D pelvic images reconstructed from preoperative CT data. Paired-point matching navigation is not very accurate in patients with severe deformities [5]. In addition, not all institutions have expensive CT-based navigation systems.

When THA is performed in the lateral decubitus position, the pelvic tilt error is large [10], and inadequate orientation of the acetabular component is a concern; however, when performed in the supine position, this error is smaller [11]. Nevertheless, the use of intraoperative fluoroscopy or X-ray to confirm alignment of the acetabular component is common. Accelerometer-based navigation systems are less expensive than CT-based navigation systems; they do not require preoperative CT, and they display the angle in real time using multiple reference points during surgery. However, the accuracy of cup orientation using accelerometer-based navigation systems remains unclear.

The purpose of this study was to compare the accuracy of cup orientation in THA performed in the supine position using CT-based and accelerometer-based navigation systems.

\section{Methods}

\section{Patients' background characteristics}

This study was approved by the Ethics Committee of the institution. This was a retrospective review of 35 hips in 35 consecutive patients (24 hips in 24 females, and 11 hips in 11 males) who underwent primary cementless THA via a modified Watson-Jones approach in the supine position (Table 1). The preoperative diagnoses were osteoarthritis for 26 hips, avascular necrosis of the femoral head for 7 hips, and rheumatoid arthritis for 2 hips. According to the Crowe classification [12], the severity of hip dysplasia in hips with osteoarthritis was defined as Crowe grade I in 33 hips and grade II in 2 hips.

\section{Devices and surgical procedure}

A CT-based paired-point matching navigation system (Vector Vision Hip CT-based version 3.5.2; BrainLab, Heimstetten, Germany) was used in this study. Preoperative CT (Discovery CT750HD; GE Medical Systems, Milwaukee, WI, USA) was performed from the pelvis to the knee. Imaging settings were as follows: tube voltage, 120 $\mathrm{kV}$; tube current, $150 \mathrm{~mA}$; slice thickness, $2 \mathrm{~mm}$; and slice pitch, $2 \mathrm{~mm}$. CT images were saved in Digital Imaging and Communications in Medicine (DICOM) format and imported into the navigation system for preoperative planning and intraoperative registration. An accelerometer-based navigation system (HipAlign Supine; OrthAlign, Aliso Viejo, CA, USA) was used in this study.

Surgery was performed by a single operator who had performed $\geq 600$ THA procedures. Both accelerometerbased navigation and CT-based navigation were used simultaneously (Fig. 1). During surgery, Schantz screws were inserted into the ala of the ilium on the affected side. A T-shaped reference antenna with three infrared reflection spheres was secured to the Schantz screws. In the registration of the CT-based navigation system, two points on the bilateral anterior superior iliac spines

Table 1 Demographic data

\begin{tabular}{|c|c|}
\hline & $n=35$ \\
\hline Age (years) ${ }^{a}$ & $65 \pm 11.2(45-85)$ \\
\hline Sex: male/female ${ }^{b}$ & $11 / 24$ \\
\hline Treated side: right/left ${ }^{b}$ & 24/11 \\
\hline \multicolumn{2}{|l|}{ Diagnosis $^{b}$} \\
\hline Osteoarthritis & 26 \\
\hline Osteonecrosis & 7 \\
\hline Rheumatoid arthritis & 2 \\
\hline Height $(m)^{a}$ & $1.56 \pm 0.1(1.39-1.77)$ \\
\hline Weight $(\mathrm{kg})^{\mathrm{a}}$ & $58.3 \pm 12(36.6-93.4)$ \\
\hline BMI $\left(\mathrm{kg} / \mathrm{m}^{2}\right)^{\mathrm{a}}$ & $23.9 \pm 3.4(15.7-31.6)$ \\
\hline Crowe G1/2/3/4 & $33 / 2 / 0 / 0$ \\
\hline Sharp angle $\left(^{\circ}\right)^{a}$ & $41.7 \pm 6.9(26-58)$ \\
\hline CE angle $\left({ }^{\circ}\right)^{a}$ & $19.9 \pm 19.9(-49-63)$ \\
\hline \multicolumn{2}{|l|}{ Surgical approach ${ }^{b}$} \\
\hline Modified Watson-Jones & 35 \\
\hline
\end{tabular}

$B M I$ body mass index, $C E$ angle center edge angle

${ }^{a}$ Values expressed as means \pm SD (range)

${ }^{b}$ Values expressed as number of patients 

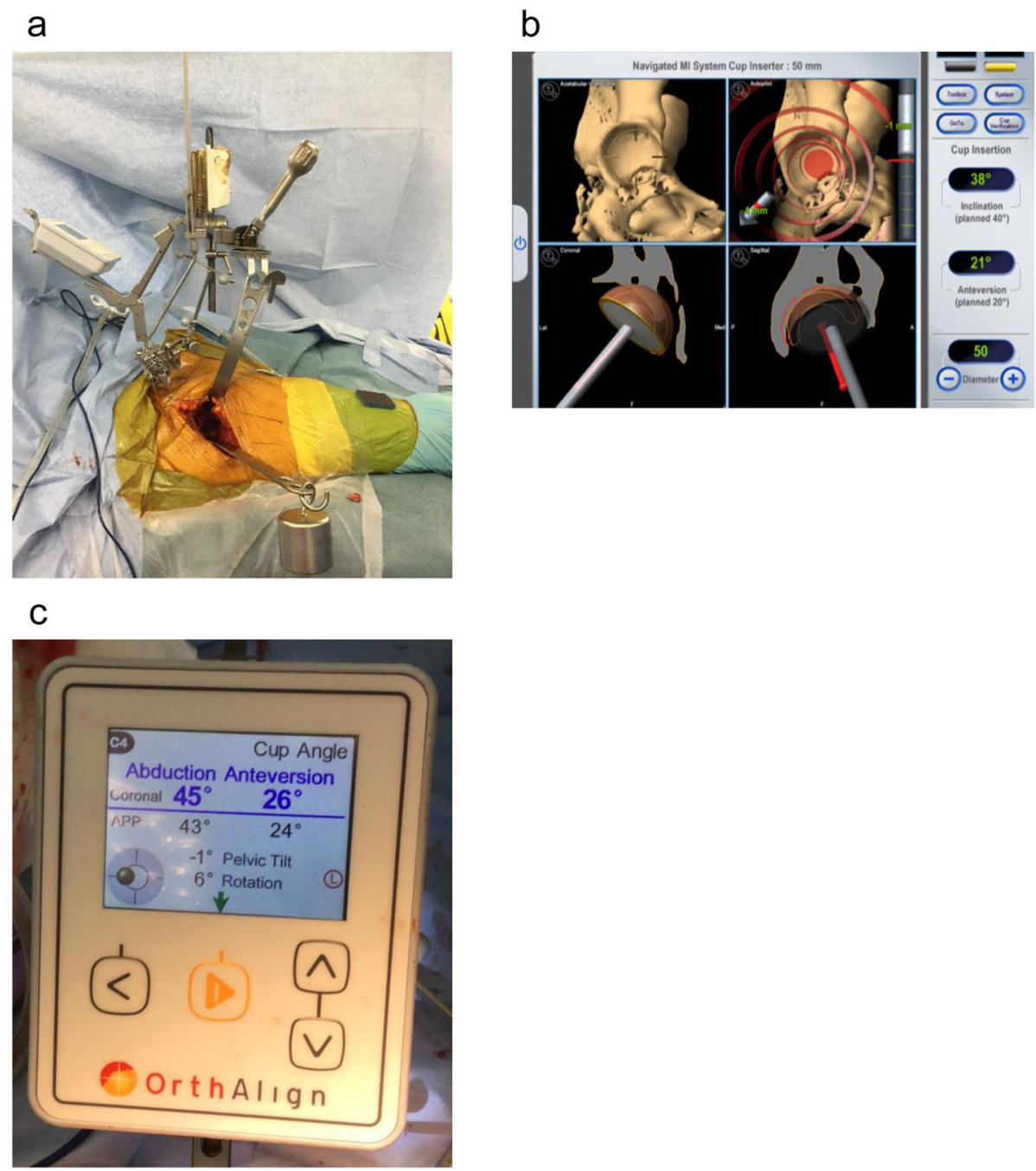

Fig. 1 Total hip arthroplasty was performed in each patient with simultaneous CT- and accelerometer-based navigation (a). Operative inclination and operative anteversion were displayed on the CT navigation system (b), and radiographic inclination and radiographic anteversion were displayed on the accelerometer-based system (c). Pelvic tilt and pelvic rotation were also displayed simultaneously on the accelerometer-based system

(ASISs), 4 points on the iliac crest, 4 points on the acetabular edge, and 7 points inside the acetabulum were acquired for registration. In the registration of the accelerometer-based navigation, the pelvic base of the accelerometer was fixed with Schantz screws. The sensor was attached, and landmark registration was performed. In table registration, the body axis was aligned with the horizontal axis. Registration of three anterior pelvic plane (APP) landmarks was performed: the bilateral ASISs and the pubic symphysis. After reaming acetabular bone, a cementless hemisphere cup (G7; Zimmer Biomet G.K., Tokyo, Japan) was placed in the reamed acetabulum in an aimed alignment using CT-based navigation. A cementless cup was fixed with three additional screws. The operator measured cup inclination and anteversion of the acetabular component by touching 5 points on its outer margin with a pointer. The operatively defined angles were displayed on the screen of the CT-based navigation (Fig. 1) [13]. The radiographically defined angles were displayed on the screen of the accelerometer-based navigation [13]. Measurements were performed three times, with mean values recorded.

\section{Evaluations}

Postoperative CT data were saved in DICOM format and imported to 3D templating software version 03.08.05 (Kyocera Medical, Kyoto, Japan). First, the pelvic coordinate system was set to the APP in the coronal, sagittal, and horizontal planes. The radiographic cup inclination angle was measured on the slice in which the diameter of the acetabular component in the coronal plane was maximal on the coronal plane (Fig. 2a) [13]. 

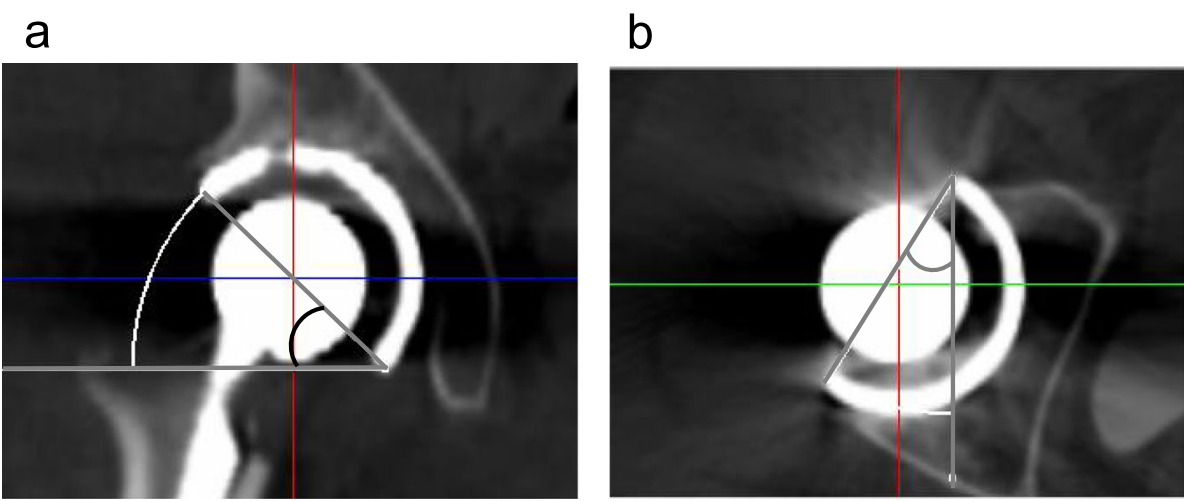

Fig. 2 Pelvic coordinate system set to the anterior pelvic plane. Measurement of radiographic inclination on the tomographic coronal plane (a) and anatomical anteversion on the tomographic axial plane (b)

The anatomical anteversion angle was similarly measured in the horizontal plane (Fig. 2b). All measurements were repeated two times each by two orthopaedic surgeons, with mean values calculated. All angles of the acetabular component were given as radiographically defined angles [13]. Accuracy of the acetabular component orientation was defined as the absolute difference between the intraoperative record and postoperative measurements on CT using either CT-based or accelerometer-based navigation [7].

The primary endpoint was to compare the accuracy of cup orientation (absolute difference between the intraoperative record and the postoperative measurement) between the two navigation systems using postoperative $\mathrm{CT}$. The secondary endpoints were intra- and postoperative complications. We also assessed the proportion of patients within the safe zone (i.e., $40^{\circ} \pm 10^{\circ}$ inclination; $15^{\circ} \pm 10^{\circ}$ anteversion) [14].

\section{Sample size and statistical analysis}

In a pilot study, the mean absolute value of the differences in postoperative measurement from the intraoperative record for cup anteversion was $3.6^{\circ}$ with CT-based navigation and $4.3^{\circ}$ with accelerometer-based navigation, with a standard deviation of $1.8^{\circ}$. The mean absolute value of the differences in postoperative measurement from the intraoperative record for cup inclination was $2.6^{\circ}$ with CTbased navigation and $3.3^{\circ}$ with accelerometer-based navigation, with a standard deviation of $1.8^{\circ}$. Based on the effect size in this pilot study, a power calculation $(p<0.05$; power 0.8 ) suggested that 33 patients would be needed for a trial. Variables with normal distribution were compared using paired $t$ tests, and variables with non-normal distribution were compared using the Wilcoxon signed-rank test. Values are shown as the mean \pm standard deviation, and $p<0.05$ was considered statistically significant. Statistical analysis was conducted using SPSS for Windows version 21 (IBM Corporation, Armonk, NY, USA).

\section{Results}

Demographic data

Patient background characteristics are presented in Table 1.

\section{Accuracy of cup orientation and complications}

The navigation systems functioned normally in all cases. Acetabular component orientation during surgery was measured at an inclination of $39.6^{\circ}$ (range $32.5^{\circ}-45.4^{\circ}$ ) and anteversion of $18.6^{\circ}$ (range $11^{\circ}-32.3^{\circ}$ ) with CT-based navigation and an inclination of $38.7^{\circ}$ (range $29^{\circ}-50^{\circ}$ ) and anteversion of $13.9^{\circ}$ (range $5^{\circ}-26^{\circ}$ ) with accelerometerbased navigation. No significant differences were observed between the CT- and accelerometer-based navigation systems in cup inclination $(p=0.16)$ (Table 2). There was a significant difference in cup anteversion between CTbased and accelerometer-based navigation $(p<0.001)$. The cup inclination on postoperative CT was $40.8^{\circ} \pm 4.1^{\circ}$ $\left(31.9^{\circ}-48.8^{\circ}\right)$, and the anteversion was $16.4^{\circ} \pm 4.0^{\circ}\left(8.6^{\circ}-\right.$ $\left.22.2^{\circ}\right)$. The absolute difference from the values measured on postoperative $\mathrm{CT}$ was $2.7^{\circ}$ inclination (range $0^{\circ}-7.2^{\circ}$ ) and $2.8^{\circ}$ anteversion (range $0^{\circ}-12^{\circ}$ ) for CT-based navigation and $3.3^{\circ}$ inclination (range $0.1^{\circ}-8.3^{\circ}$ ) and $3.4^{\circ}$ anteversion (range $0.3^{\circ}-8.5^{\circ}$ ) for accelerometer-based navigation. The differences from the postoperatively measured values in inclination and anteversion measured with the two systems were not significant $(p=0.29,0.34$, respectively, Table 2). The proportion of patients within the safe zone was $100 \%$ (35/35 patients, Fig. 3). Loosening of Schantz screws that were used for fixation was not observed. No patients showed postoperative dislocation, fracture, or required repeated surgery for other reasons.

\section{Discussion}

In this study, we performed primary cementless THA in the supine position using CT- and accelerometer-based navigation simultaneously and compared the accuracy of cup orientation between the two systems. The results 
Table 2 Intraoperative record and absolute value of differences in postoperative measurement from the intraoperative record for cup angle

\begin{tabular}{lllll}
\hline & & CT-based navigation group $(n=35)$ & Accelerometer-based navigation group $(n=35)$ & $p$ value \\
\hline Intraoperative angles $^{\mathrm{a}}$ & Inclination $\left(^{\circ}\right)$ & $39.6 \pm 3.4(32.5-45.4)$ & $38.7 \pm 5.0(29-50)$ & $0.1565^{\mathrm{b}}$ \\
& Anteversion $\left(^{\circ}\right)$ & $18.6 \pm 4.7(11-32.3)$ & $13.9 \pm 5.0(5.0-26)$ & $0.0000^{\mathrm{b}}$ \\
Absolute value of differences $^{\mathrm{a}}{ }^{\mathrm{C}}$ & Inclination $\left(^{\circ}\right)$ & $2.7 \pm 2.0(0-7.2)$ & $3.3 \pm 2.4(0.1-8.3)$ & $0.2878^{\mathrm{b}}$ \\
& Anteversion $\left(^{\circ}\right)$ & $2.8 \pm 2.6(0-12)$ & $3.4 \pm 2.2(0.3-8.5)$ & $0.3366^{\mathrm{b}}$ \\
\hline
\end{tabular}

Values expressed as means \pm SD (range)

${ }^{\mathrm{b}}$ Paired $t$ test

confirmed that acetabular cup inclination and anteversion were measured with equivalent accuracy using CTand accelerometer-based navigation.

CT-based navigation is useful for positioning implants in the correct alignment and orientation during THA, and newer navigation systems enable more accurate implant positioning [15]. CT-based navigation can help achieve correct cup orientation irrespective of operator experience [16]. The accuracy of CT-based navigation is such that the absolute error of inclination and anteversion is within approximately $4^{\circ}$, even in patients with severe deformity [5]. In this study, the absolute error of both inclination and anteversion measured with CTbased navigation was $<3^{\circ}$. However, CT-based navigation requires radiation exposure in addition to that from preoperative $\mathrm{CT}$, with increased cost.

Cup malposition, with respect to the Lewinnek safe zone, is an independent risk factor for postoperative dislocation (odds ratio 1.88) [17]. Because the safe zone for cup anteversion is narrower than that for inclination [4], the angle of anteversion must be carefully determined. When THA is performed in the supine position, cup

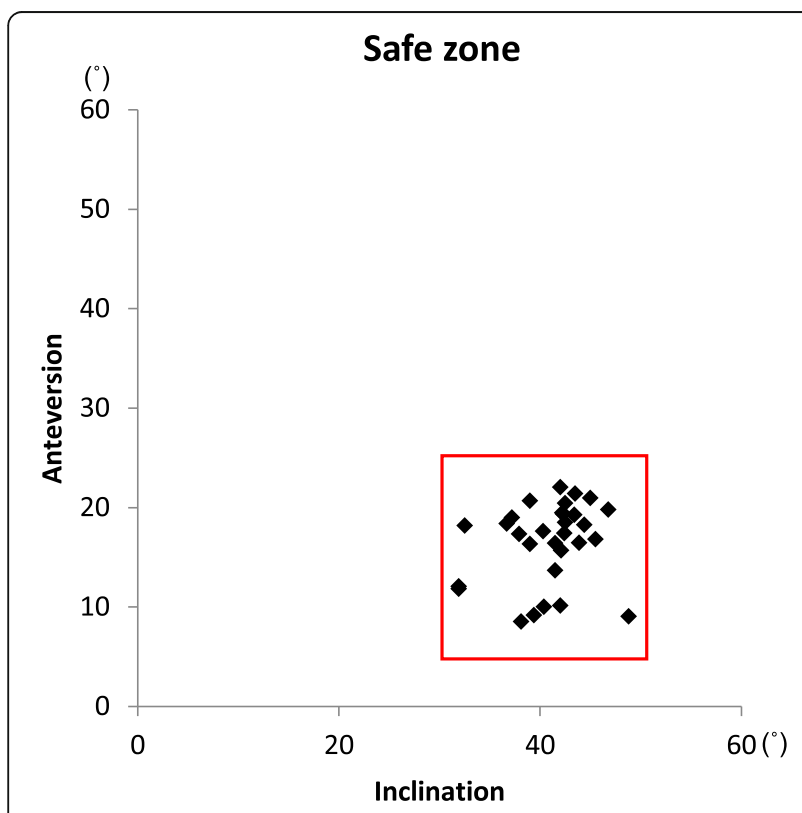

Fig. 3 Patients within the safe zone alignment can be monitored with the use of intraoperative fluoroscopy or X-ray. However, surgery should ideally be performed without the use of intraoperative radiation. A factor affecting measurement accuracy using conventional mechanical guidance for THA in the supine position is the variation in pelvic tilt and rotation during surgery compared with the preoperative measurements. Kanazawa et al. [18] reported approximately $3^{\circ}$ of pelvic movement in the sagittal, horizontal, and coronal planes during cup positioning. When THA is performed using the HipAlign Supine system, preregistration is performed before the acetabular component is positioned. Because the accelerator within the unit is constantly working, the pelvis is continuously traced. The system displays tilt and rotation of the pelvis with respect to the direction of gravity during surgery, making it more accurate than mechanical guides are, as the accelerometer is unaffected by changes in pelvic tilt during cup positioning [18].

The value of imageless navigation in avoiding radiation exposure has been reported $[7,19]$. The mean errors in inclination and cup anteversion with imageless navigation in THA were $6.8^{\circ}$ and $3.7^{\circ}$, respectively [15]. Kalteis et al. [19] reported that for patients with anatomical abnormalities such as acetabular dysplasia, imageless navigation imparted a number of disadvantages compared with CT-based navigation. The present study found that in THA performed using the HipAlign Supine system, both inclination and anteversion were highly accurate with no radiation exposure or expensive systems. The HipAlign Supine system has some advantages. Because this system is not optical, the surgeon does not need to worry about his/her positioning. The angle displayed meets the radiographic definition according to both APP and functional pelvic plane (FPP) criteria, even without preoperative $\mathrm{CT}$ data.

Although for many years, the APP was considered to be a global reference, it is subject to significant interindividual variations and variations during positional changes [20]. Reclining the pelvis by $1^{\circ}$ leads to a change in functional anteversion of approximately $0.7^{\circ}$ [21]. Accordingly, the level of preoperative tilt of the APP must be considered to correctly determine the cup orientation angle [22]. The FPP has been proposed as an alternative 
to the APP that takes functional pelvic tilt into account during THA [23]. The acetabular component orientation may be adjusted according to pelvic tilt at the time of cup insertion [24-26]. In this study, however, we used the APP rather than the FPP to compare the accuracy between the two navigation systems. This method was used because during surgery, tilt, rotation, and other pelvic parameters should be the same as they are during radiographic assessment. However, the mean intraoperative anterior pelvic tilt was $1.4^{\circ}$, with a mean internal rotation of $1.4^{\circ}$ and a mean adduction of $0.9^{\circ}$ with the patient in the supine position [11]. A difference in pelvic tilt makes it impossible to perform accurate comparisons of cup alignment on preoperative and postoperative CT images. We therefore used the APP rather than the FPP as the standard plane in this study.

Our study had limitations. Surgery was performed using CT-based navigation as the reference for cup orientation, which was within the safe zone for all patients. However, because we used accelerometer-based and CT-based navigation simultaneously in the same patients, we were able to properly compare accuracy. Second, most patients in this study had Crowe type I or II, with only mild deformity. Severe deformity reportedly reduces the accuracy of CT-based navigation [6]. A study performed in patients with more severe deformity might have obtained a different result. Finally, most patients were comparatively thin, with a mean body mass index of $23.9 \mathrm{~kg} / \mathrm{m}^{2}$. Factors associated with inadequate cup orientation include the surgical approach; the surgeon's level of experience; a clumsy, low-intervention surgical approach; and obesity [27]. Axial digitization errors affect the anteversion angle by $1.8^{\circ}$ for the ipsilateral ASIS, $4.4^{\circ}$ for the contralateral ASIS, and $6.8^{\circ}$ for the centre of the two pubic tubercles [28]. Pubis registration error thus has a major effect on cup orientation accuracy. An investigation including obese patients might have obtained different results.

\section{Conclusions}

The absolute difference in cup anteversion measured with accelerometer-based navigation was within acceptable limits. The HipAlign Supine provides potential benefit in reducing cup alignment error during THA in the supine position, and its simplicity may contribute to the wider use of computer-assisted orthopaedic surgery.

\footnotetext{
Abbreviations

THA: Total hip arthroplasty; CT: Computed tomography; 2D: 2-dimensional; DICOM: Digital imaging and communications in medicine; ASIS: Anterior superior iliac spine; APP: Anterior pelvic plane; FPP: Functional pelvic plane
}

\section{Acknowledgements}

We would like to thank Editage (www.editage.com) for English language editing.

\section{Authors' contributions}

$\Pi 1$ performed the surgeries and contributed to the study design, the writing of the paper, and drafting of the manuscript. $\Pi 2$ participated in the design of the study. KY, TS, and YK collected and analyzed the data. TO reviewed and edited the manuscript. All authors read and approved the final manuscript.

\section{Funding}

None.

\section{Availability of data and materials}

All data used and analysed during this study are available from the corresponding author upon reasonable request.

Ethics approval and consent to participate

This research was approved by the ethics committee of the Okayama University.

Consent for publication

Not applicable.

\section{Competing interests}

The authors declare that they have no competing interests.

\section{Author details}

'Department of Orthopaedic Surgery, Okayama University Hospital, 2-5-1 Shikata-cho, Kitaku, Okayama 700-8558, Japan. ${ }^{2}$ Department of Medical Materials for Musculoskeletal Reconstruction, Okayama University Graduate School of Medicine, Dentistry and Pharmaceutical Sciences, 2-5-1

Shikata-cho, Kitaku, Okayama 700-8558, Japan.

Received: 14 December 2019 Accepted: 3 April 2020

Published online: 15 April 2020

\section{References}

1. McCarthy TF, Alipit V, Nevelos J, et al. Acetabular cup anteversion and inclination in hip range of motion to impingement. J Arthroplast. 2016;31(9 Suppl):264-8

2. Kennedy JG, Rogers WB, Soffe KE, et al. Effect of acetabular component orientation on recurrent dislocation, pelvic osteolysis, polyethylene wear, and component migration. J Arthroplast. 1998;13(5):530-4.

3. Teeter MG, Goyal P, Yuan X, et al. Change in acetabular cup orientation from supine to standing position and its effect on wear of highly crosslinked polyethylene. J Arthroplast. 2018;33(1):263-7.

4. Murphy WS, Yun $\mathrm{HH}$, Hayden B, et al. The safe zone range for cup anteversion is narrower than for inclination in THA. Clin Orthop Relat Res. 2018;476(2):325-35.

5. Yamada $K$, Endo $H$, Tetsunaga $T$, et al. Accuracy of cup positioning with the computed tomography-based two-dimensional to three-dimensional matched navigation system: a prospective, randomized controlled study. J Arthroplast. 2018;33(1):136-43.

6. Tsuda K, Haraguchi K, Koyanagi J, et al. A forty millimetre head significantly improves range of motion compared with a twenty eight millimetre head in total hip arthroplasty using a computed tomography-based navigation system. Int Orthop. 2016;40(10):2031-9.

7. Lass R, Kubista B, Olischar B, et al. Total hip arthroplasty using imageless computer-assisted hip navigation: a prospective randomized study. J Arthroplast. 2014:29(4):786-91.

8. Sugano N. Computer-assisted orthopaedic surgery and robotic surgery in total hip arthroplasty. Clin Orthop Surg. 2013;5(1):1-9.

9. Sugano N, Nishii T, Miki H, et al. Mid-term results of cementless total hip replacement using a ceramic-on-ceramic bearing with and without computer navigation. J Bone Joint Surg (Br). 2007;89(4):455-60.

10. Tetsunaga $T$, Yamada $K$, Tetsunaga $T$, et al. Comparison of the accuracy of CT- and accelerometer-based navigation systems for cup orientation in total hip arthroplasty. Hip Int. 2020;10.1177/1120700020904940: 1120700020904940

11. Grammatopoulos G, Gofton W, Cochran M, et al. Pelvic positioning in the supine position leads to more consistent orientation of the acetabular component after total hip arthroplasty. Bone Joint J. 2018;100-b(10):1280-8. 
12. Crowe JF, Mani VJ, Ranawat CS. Total hip replacement in congenital dislocation and dysplasia of the hip. J Bone Joint Surg Am. 1979;61(1):15-23.

13. Murray DW. The definition and measurement of acetabular orientation. J Bone Joint Surg (Br). 1993;75(2):228-32.

14. Lewinnek GE, Lewis JL, Tarr R, et al. Dislocations after total hip-replacement arthroplasties. J Bone Joint Surg Am. 1978;60(2):217-20.

15. Nakahara I, Kyo T, Kuroda Y, et al. Effect of improved navigation performance on the accuracy of implant placement in total hip arthroplasty with a CT-based navigation system. J Artif Organs. 2018;21(3):340-7.

16. Iwana D, Nakamura N, Miki H, et al. Accuracy of angle and position of the cup using computed tomography-based navigation systems in total hip arthroplasty. Comput Aided Surg. 2013;18(5-6):187-94.

17. Danoff JR, Bobman JT, Cunn G, et al. Redefining the acetabular component safe zone for posterior approach total hip arthroplasty. J Arthroplast. 2016; 31(2):506-11.

18. Kanazawa M, Nakashima $Y$, Ohishi M, et al. Pelvic tilt and movement during total hip arthroplasty in the lateral decubitus position. Mod Rheumatol. 2016;26(3):435-40.

19. Kalteis $T$, Handel $M$, Bathis $H$, et al. Imageless navigation for insertion of the acetabular component in total hip arthroplasty: is it as accurate as CT-based navigation? J Bone Joint Surg (Br). 2006;88(2):163-7.

20. Barbier $\mathrm{O}$, Skalli W, Mainard $\mathrm{L}$, et al. The reliability of the anterior pelvic plane for computer navigated acetabular component placement during total hip arthroplasty: prospective study with the EOS imaging system. Orthop Traumatol Surg Res. 2014;100(6 Suppl):S287-91.

21. Muller O, Lembeck B, Reize $P$, et al. Quantification and visualization of the influence of pelvic tilt upon measurement of acetabular inclination and anteversion. Z Orthop Ihre Grenzgeb. 2005;143(1):72-8.

22. Beckmann J, Luring C, Tingart M, et al. Cup positioning in THA: current status and pitfalls. A systematic evaluation of the literature. Arch Orthop Trauma Surg. 2009;129(7):863-72.

23. Pierrepont J, Hawdon G, Miles BP, et al. Variation in functional pelvic tilt in patients undergoing total hip arthroplasty. Bone Joint J. 2017;99-b(2):184-91.

24. Babisch JW, Layher F, Amiot LP. The rationale for tilt-adjusted acetabular cup navigation. J Bone Joint Surg Am. 2008;90(2):357-65.

25. Lembeck B, Mueller $\mathrm{O}$, Reize $\mathrm{P}$, et al. Pelvic tilt makes acetabular cup navigation inaccurate. Acta Orthop. 2005;76(4):517-23.

26. Maratt JD, Esposito Cl, McLawhorn AS, et al. Pelvic tilt in patients undergoing total hip arthroplasty: when does it matter? J Arthroplast. 2015; 30(3):387-91.

27. Callanan MC, Jarrett B, Bragdon CR, et al. The John Charnley Award: risk factors for cup malpositioning: quality improvement through a joint registry at a tertiary hospital. Clin Orthop Relat Res. 2011;469(2):319-29.

28. Lee YS, Yoon TR. Error in acetabular socket alignment due to the thick anterior pelvic soft tissues. J Arthroplast. 2008;23(5):699-706.

\section{Publisher's Note}

Springer Nature remains neutral with regard to jurisdictional claims in published maps and institutional affiliations.

Ready to submit your research? Choose BMC and benefit from:

- fast, convenient online submission

- thorough peer review by experienced researchers in your field

- rapid publication on acceptance

- support for research data, including large and complex data types

- gold Open Access which fosters wider collaboration and increased citations

- maximum visibility for your research: over $100 \mathrm{M}$ website views per year

At BMC, research is always in progress.

Learn more biomedcentral.com/submissions 